\title{
Editorial
}

\section{COVID-19 and Anti-Asian Racism}

\author{
Alexandra Chang \\ Associate Professor, Department of Arts, Culture and Media, \\ Rutgers University-Newark, Newark, NJ, USA \\ alexa.chang@rutgers.edu \\ Alice Ming WaiJim \\ Professor, Department of Art History, Concordia University, \\ Montreal, Quebec, Canada \\ alice.jim@concordia.ca
}

This issue of ADVA responds to the COVID-19 pandemic and the resulting highly-mediatized surge in anti-Asian racism and misogyny, which has exacerbated deeply-rooted anti-Asian Pacific racisms in North America and underlined continuing legacies of global histories of colonialism and empire. In order to hold space for collective grief, anger, frustration, and exhaustion, and to address the heightened sense of precarity we are experiencing, many of the contributions to this issue focused on how the pandemic affected Asian diasporic artists, activists, community organizers, curators, and scholars this past year.

Our authors and editors were not immune to the toll of the pandemic. We were affected by illness, from covid or from pre-existing conditions, worsened by the overburdened healthcare system and global strain, first in the search for a vaccine and now in its administration and dispensation. Rather than rush back to a business-as-usual model, we extended our deadlines and engaged with slowness; we practiced a politics of refusal. Our emergency editorial 
meeting to ascertain the impact of the pandemic on the journal and its production schedule conveyed to us that many of our authors, who tend to be of Asian descent, were burning out because of the increased burden of representation, of self-presenting, with many simultaneously pivoting to online teaching while dealing with racism and mounting demands for equity and diversity work. This additional work, already traditionally shouldered by POC, has been further spurred by the global racial reckoning as institutions try to give themselves instant overhauls at least on paper-stopgap measures that don't move beyond the PR patchwork—-rather than the real work of dismantling systemic racism.

The offerings of this issue range from pieces solicited just weeks to months into the pandemic, in solidarity with Black Lives Matter and concurrent to the US presidential race and post-vaccine discussions as the pandemic held sway into its second year. All the organizing work-from ad-hoc talks to global protests and campaigns - matters and is reflected in the range of formats of this special issue, which includes roundtables, Q\&A, vignettes, resources, alongside our usual sections. In this way, we chose to showcase multiple voices and the immediacy of these important discussions rather than single-authored academic production. Meanwhile, our one peer-reviewed article by Dipti Desai, "There Was So Much Silence Growing Up...: Artistic Interventions of Tomie Arai and Flo Oy Wong," reflects on the artists' work with community-based oral histories to unearth ways in which historical anti-Asian US immigration policies have had lasting effects on communities.

What became evident was on the one hand, the need to focus on allyshiprecognizing the historic solidarities between Asian and Black communities in the diaspora and the ongoing relations with Indigenous peoples-and, on the other, the re-emergence of anti-Asian racism and Yellow Perilist discourses, and the ongoing racial justice activism. The first curated artist pages feature work by Montreal-based artist Chun Hua Catherine Dong, who illuminates entanglements of social media, technology, race, and the diasporic body during and beyond the time of the pandemic. The LACMA, GYOPO, and StopDiscriminAsian-organized roundtable "Racism Is a Public Health Issue: Addressing Prejudices against Asian Americans during the covid19 Pandemic" with Christine Y. Kim, Jeff Chang, Cathy Park Hong, Russell Jeung, Bowen Yang, and Anicka Yi, moderated by Kibum Kim, is an example of the conversations that were held to underline historic and widespread systemic racism and to forward the need for solidarity and push against divides among POC communities. The roundtable discussion with six members of the godzilla eleven-Tomie Arai, Todd Ayoung, Shelly Bahl, Kerri Sakamoto, Lynne Yamamoto and ADVA's Alexandra Chang-further emphasizes the importance 
of cross-community coalition building as well as collective care and mutual aid. This discussion extended their thoughts from the pandemic to questioning what the responsibility of care can look like for institutions and the communities they serve.

Organized in solidarity with Black Lives Matter and global movements denouncing systems of oppression, racism, and police brutality, the panel discussion "Hyper(in)visibility," curated by stephanie mei huang, brought together artists Pearl C. Hsiung, Maia Ruth Lee, Astria Suparak, Hồng-Ân Trương, and Christine Tien Wang to reflect upon their practices, reckoning with the racialized and gendered spectrum of visibility.

Examining the impacts of COVID-19 on the contemporary art world, in their Q\&A feature, art historians Kanwal Syed and Varda Nisar discuss with New York-based artist Ruby Chishti the experience of having her solo exhibition at Aicon Gallery shut down to comply with early lockdown procedures in 2020. Foregrounding the artist's body of work, her experience moving from Pakistan to New York in the early 200os, and her feminist approach to issues of diaspora, trauma, and ecocriticism, the discussion illuminates the potential challenges facing diasporic women artists in a post-pandemic world.

This issue's Spotlight feature is written by US Area Editor Emily Hue and showcases four US-based outreach projects mobilized during the pandemic in the spirit of solidarity and social justice. The projects reflect the many ways that Asian American Pacific Islander communities have worked to connect and care for each other, documenting shared anxieties, struggles, and grief, as well as moments of joy and catharsis. During the first summer of the pandemic in 2020, the Ethnocultural Art Histories Research Group (EAHR) based at Concordia University, curated the Hear Us Now! Instagram Project (@eahrconcordia) with the goals of connecting and supporting work by Black, Indigenous, and other racialized visible minority artists. The curated pages features a selection of work by five Asian Canadian artists from this online social media project: My-Van Dam, Ivetta Sunyoung Kang, Reyhan Yazdani, Florence Yee, and Ketty Zhang. Small to large initiatives all play their parts.

Entwined with the double pandemic of CoviD-19 and racism, is the Eco Crisis, which is the focus of the next double-issue edited by Alexandra Chang. The special issue presents the work of artists including a Q\&A with Jane Chang Mi and Greg Dvorak; May Joseph in conversation with Jaimey Hamilton-Farris; the Diasporic Asian Art Network College Art Association roundtable with Sue Huang, Jean Shin, and Mary Ting; and artist pages on Katherine Behar and Gaye Chan; as well as articles on Sean Connelly and Patty Chang. It will also include a Perspectives piece in memoriam of the great feminist art historian and a fervent supporter of artists of Asian descent, Moira Roth, who authored 
an interview with artist Dinh Q. Lê for our inaugural issue. With volume 6, Alexandra Chang, ADVA's co-founding editor-in-chief since 2014 will be stepping down from this important role. She joins Canadian poet, scholar, editor, and activist Roy Miki (Board of Advisors, 2015-2020) as ADVA emeriti. Finally, with this issue, we welcome our new Managing Editor, Victoria Nolte, who has been indispensable in getting us back on track. Stay with us as we weather through these tumultuous times, in solidarity in caring for all our relations. 